\title{
Towards a Better Understanding of Relationship between Preisach Densities and Polarization Reversals on Hysteresis Characteristic
}

\author{
Teerawat Monnor, ${ }^{1}$ Yongyut Laosiritaworn, ${ }^{2}$ and Rattikorn Yimnirun ${ }^{1}$ \\ ${ }^{1}$ School of Physics, Institute of Science, Suranaree University of Technology, Nakhon Ratchasima 30000, Thailand \\ ${ }^{2}$ Department of Physics and Materials Science, Faculty of Science, Chiang Mai University, \\ Chiang Mai 50200, Thailand
}

Correspondence should be addressed to Yongyut Laosiritaworn; yongyut_laosiritaworn@yahoo.com

Received 31 May 2013; Revised 17 August 2013; Accepted 19 August 2013

Academic Editor: Charles Rosenblatt

Copyright (C) 2013 Teerawat Monnor et al. This is an open access article distributed under the Creative Commons Attribution License, which permits unrestricted use, distribution, and reproduction in any medium, provided the original work is properly cited.

This work performed Preisach modeling on hysteresis loops, where the contributions of Preisach density characteristics on hysteresis reversals were investigated. Specifically, the three-dimensional Gaussian-distribution function was used to construct the Preisach densities for extracting the associated hysteresis loops. In particular, the influences of three key Gaussian Preisach density characteristics (i.e., density sharpness, density center, and splitting densities) on hysteresis behavior were examined. It was found that sharper density induces more harmonized domain switching, so polarization derivative is enhanced at the coercivity, while the density center indicates the range of external field at which most switching occurs. Moreover, the splitting of the density was found to represent pinching in hysteresis loops, where material tends to actively respond in two different ranges of external field. Consequently, based on these results, significant hysteresis behavior can be revealed using minimal parameters via appropriate mathematical function; that is, another step enhances the fundamental understanding in the hysteresis topic using the Preisach framework.

\section{Introduction}

The hysteresis loop (HL) has long been known to be one of the key characteristics specifying how materials respond to dynamic external perturbations (e.g., electric field, magnetic field, or stress). As a result, the HL has been intensively studied, particularly in ferroelectric and ferromagnetic materials. This is for enhancing functionalities of electronic devices, for example, ferroelectric relaxors [1] and ferroelectric random access memory (FeRAM) [2]. Therefore, being a supportive ground for experiments in enhancing the technological development, efficient theoretical tools are crucial. One of these theoretical tools in the understanding of hysteresis phenomena is the Preisach model (PM) [3], which has been employed to investigate key characteristics of HL, for example, contributions of reversible and irreversible polarization [4] and pinching effect [5].
One of the well-known schemes for investigating hysteresis is the first-order reversal curves (FORC) [6, 7]. Recently, the FORC studies gain success in presenting influences of experimental factors to the hysteresis phenomena. For instance, the effect of grain-size variation in $\mathrm{BaTiO}_{3}$ ceramics was encoded on the FORC distribution, where two narrow and strong peaks on the distribution represent pinching in large-grained samples [8].

Although the compatibility between the FORC distribution and the classical PM is still ambiguous, the HL modeling based on the conventional PM concept can help shading more light on macroscopic properties of the FORC. Recently, there were attempts to reveal the fundamental principles behind the FORC distributions by studying ferromagnetic nanowire arrays $[9,10]$. For instance, the hysteresis characteristic was investigated where different roles between the classical PM and the FORC distribution on describing magnetic switching 


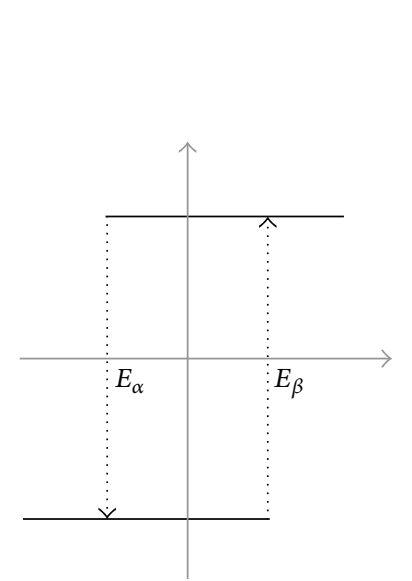

(a)

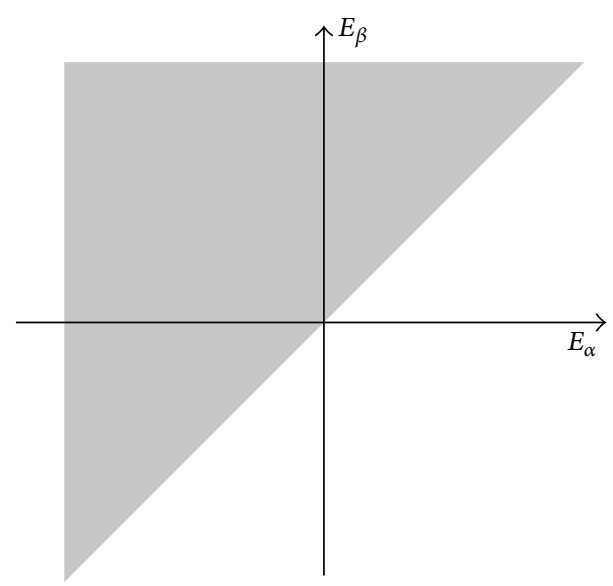

(b)

FIGURE 1: Illustrations of (a) relay operator as a hysteron operating in the Preisach model and (b) Preisach plane where all hysterons are plotted on the $E_{\beta} \geq E_{\alpha}$ area (labeled in gray).

were pointed out [10]. Specifically, the FORC concentrates on the evolution of the hysteresis switching of domains over time via two important field components, that is, the interaction field distribution and the coercive field distribution. On the other hand, the classical PM focuses on the global hysteresis behavior at steady state. Therefore, with different origins, to directly represent/relate the FORC in terms of classical hysteron is not trivial. Consequently, there are still areas for enhancing fundamental hysteresis understanding by merging main features of FORC distribution with classical PM principle to explain switching phenomena in major fractions of complex inhomogeneous materials with dynamic signatures.

Therefore, with a concentration on the Preisach density (PD) in classical PM, this work aims to illustrate the relationships between the features of the PD and the reversal behavior of hysteresis signals. The Gaussian distribution was chosen to model the PD. This is as the Gaussian function can be fine tuning using only a few parameters, that is, the position of the peak and the peak height and width (the standard deviation). These distribution functions can then shape the PD and the associated HL. Further, with the established correlations between the main characteristic between the PDs and the HLs, one can model or reconstruct the experimental hysteresis loops with further understanding in structureproperty relationship as well as providing relevant supports for experiments.

It is also important to note that there might be some limitations on implementing the classical PM to describe some physical systems. For example, as the classical PM is designed for modeling counterclockwise HL, some modifications on Preisach plane are therefore needed to model clockwise HL, for example, the capacitance-voltage $\mathrm{HL}$ of PZT-ZnO [11]. For instance, for each hysteron with switching-up field $E_{\beta}$ and switching-down field $E_{\alpha}$ (e.g., see Figure 1(a)) located on the Preisach plane (e.g., see Figure 1(b)), the plane has to be redefined from having $E_{\beta} \geq E_{\alpha}$ to $E_{\beta} \leq E_{\alpha}$ in order to model the clockwise HL signals. However, this classical PM is still very useful as it reduces degrees of freedom (on the Preisach plane) in the modeling and has been proved to yield macroscopic hysteresis reversal properties. Further, as typical experimental hysteresis is counterclockwise, by focusing on counterclockwise HL in this work, we can therefore take the advantages of the classical PM principles to describe the majority of hysteresis behaviors.

To outline, this paper is divided into 5 sections. Section 1 introduces motivation of this work and backgrounds of PM, $\mathrm{PD}$, and the use of Gaussian function. Section 2 is devoted to principles of the PM. Later in Section 3, the modification of the PD with Gaussian distribution is presented. Then, how the Gaussian-defined PD governs hysteresis features is discussed in Section 4. Finally, all the results are concluded in the conclusion, Section 5. Moreover, it should be noted that although the terminologies used were from ferroelectric language, the finding of this work is applicable to other systems where hysteresis presents as ferromagnetic or ferroelastic materials.

\section{Preisach Model}

Preisach model (PM) [3] is one of the potential tools in hysteresis modeling. For instance, it has been recognized as a significant tool for examining nonlinear response [12] and illustrating the states of ferroelectric system [6]. A major reason that makes the PM-based approaches widely employed is that the model is simple but effective in characterizing $\mathrm{HL}$ signals.

The PM can be performed by integrating the contribution of relay operators in a particular field interval. Each of the operators is termed as "hysteron" (see Figure 1(a)) which comprises bistable states at threshold fields of $E_{\beta}$ and $E_{\alpha}$ for switching to upper and to lower states, respectively. Based on a macroscopic counterclockwise reversal of $\mathrm{HL}$, 


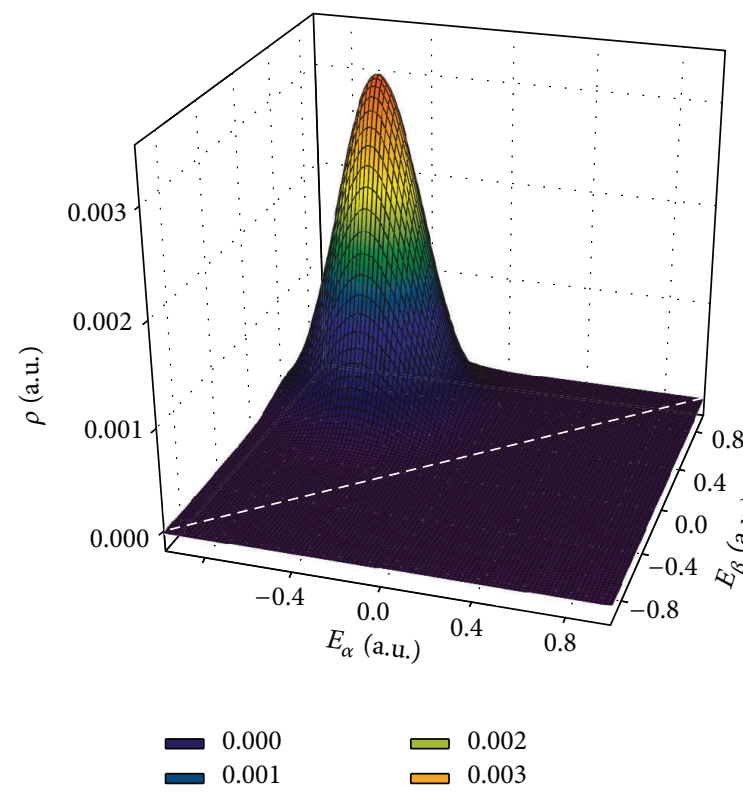

(a)
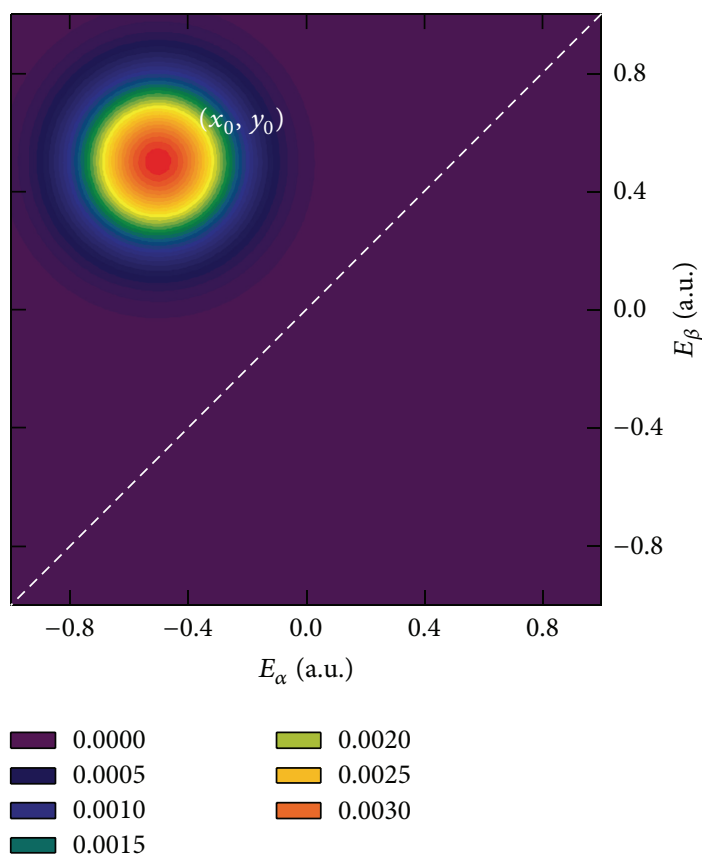

0.0030

(b)

Figure 2: Illustrations of (a) Gaussian-defined Preisach density and (b) contour plot of the three-dimensional density in (a), where $\left(x_{0}, y_{0}\right)$ indicates a center of the density.

all hysterons are defined on the so-called Preisach plane, where the upswitching field happens at threshold larger or equal to the downswitching field (i.e., $E_{\alpha} \leq E_{\beta}$ ) as shown in Figure 1(b). In addition, for the plane with discrete hysterons scattered uniformly throughout the Preisach plane, the difference between switching-up and switching-down states can be denoted here as $\mu$ represents the contribution of each hysteron on HL.

For a particular hysteresis loop, an increasing field drives materials to the up-switching state, while a decreasing field results in the opposite way. With the PM, the fields increasing and decreasing in a hysteresis measurement are represented by the increase in $E_{\beta}$-axis and the decrease in $E_{\alpha}$-axis on the Preisach plane, respectively. For a ferroelectric system, the polarization at time $t$, that is, $P(t)$, can be expressed as follows:

$$
P(t)=\iint \rho\left(E_{\alpha}, E_{\beta}\right) \varphi_{E_{\alpha}, E_{\beta}}[E(t)] d E_{\alpha} d E_{\beta} .
$$

In (1), $E(t)$ represents a time-dependent electric field, $\varphi_{E_{\alpha}, E_{\beta}}[E(t)]$ represents the Preisach operator, and $\rho\left(E_{\alpha}, E_{\beta}\right)$ represents the Preisach density. Also, the PD can be considered as a distribution of hysteresis coercivity as each hysteron can be illustrated as a component HL with different coercivity [13]. In this work, attention has been paid to the symmetric hysteresis loops, where the hysteron weight distribution, that is, $\mu\left(E_{\alpha}, E_{\beta}\right)$, was considered as the PD. Then, the $P$ $E$ loops were calculated form the cumulative contribution of $\mu\left(E_{\alpha}, E_{\beta}\right)$ in $E_{\beta}$-axis and $E_{\alpha}$-axis for increasing and decreasing fields, respectively.

\section{Modification of Preisach Density}

In order to investigate the contributions of $\mathrm{PD}$ traits on HL features, the PD in this work was defined from the three-dimensional Gaussian distribution as (e.g., see Figure 2(a))

$$
\mu\left(E_{\alpha}, E_{\beta}\right)=\frac{1}{\sqrt{2 \pi \sigma^{2}}} \exp \left[\left(\frac{E_{\alpha}-x_{0}}{\sigma}\right)^{2}+\left(\frac{E_{\beta}-y_{0}}{\sigma}\right)^{2}\right],
$$

where $\left(x_{0}, y_{0}\right)$ is the Gaussian peak position which is the "center of mass" of all hysterons projected on the Preisach plane (e.g., see Figure 2(b)) and $\sigma$ is a standard deviation governing the PD sharpness and broadness. As this work aims to examine the relations between hysteresis reversals and the PD characteristic, three scenarios of the PD have been considered: (1) variation of the PD sharpness/broadness $(\sigma)$, (2) location of the PD center $\left(x_{0}, y_{0}\right)$, and (3) splitting pattern of the PD. Note that, with the Gaussian-PD shape considered, this work focuses mainly on irreversible contributions of the hysteron on hysteresis behavior.

\section{Results and Discussions}

4.1. Variation of the PD Sharpness. To investigate the contributions of the PD sharpness/broadness, $\sigma$ in (2) was varied as shown, for example, in Figures 3(a)-3(c). It was found that an 

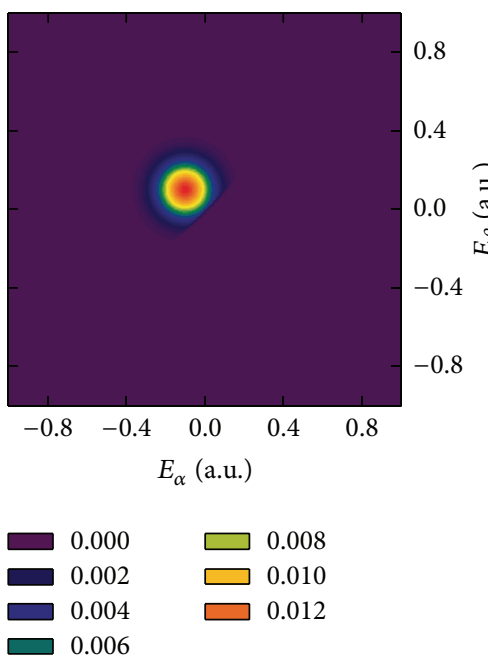

(a)

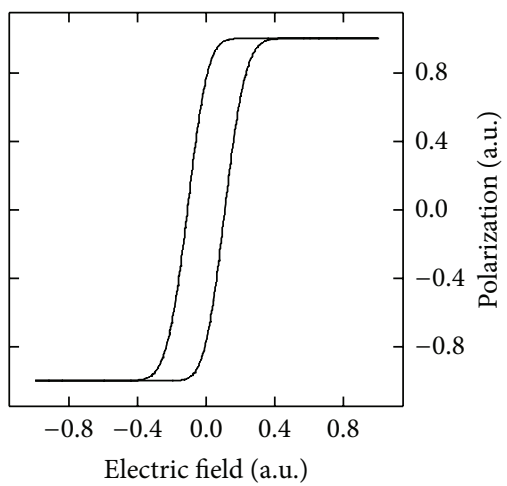

(d)

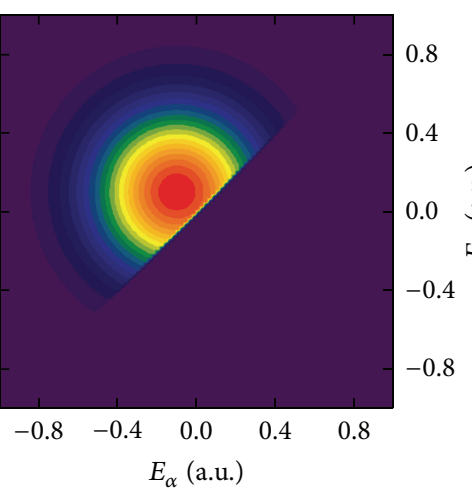

$\begin{array}{lll}0.0000 & \\ 0.0005 & \\ 0.0010 & & 0.0020\end{array}$

(b)

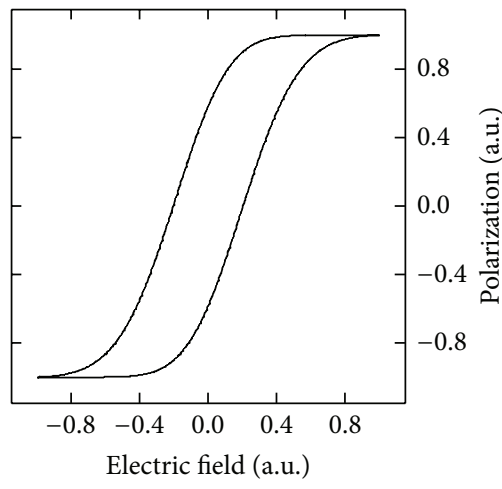

(e)

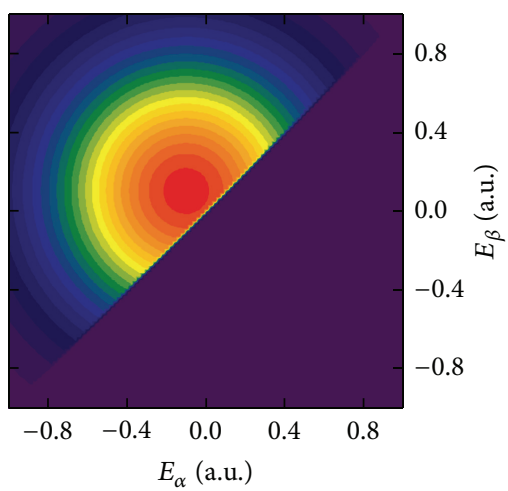

$\begin{array}{lll}0.0000 & \square & 0.0006 \\ 0.0002 & \square & 0.0008 \\ 0.0004 & & \end{array}$

(c)

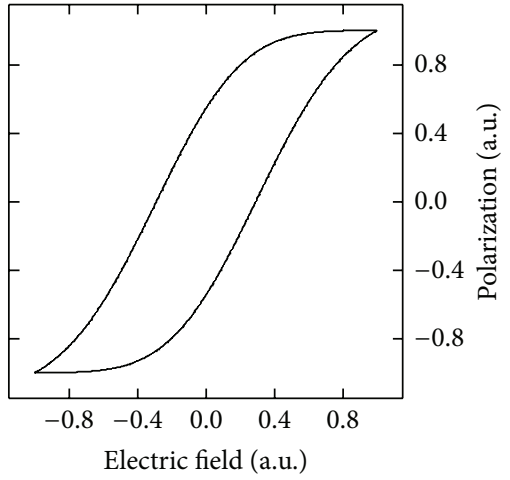

(f)

Figure 3: Gaussian-defined Preisach densities with centers located at $(-0.1,0.1)$ and width $\sigma=(\mathrm{a}) 0.10$, (b) 0.30 , and (c) 0.50 . Their associated hysteresis loops are shown in (d), (e), and (f), respectively.

increase in $\sigma$ results in sharper PD (e.g., in Figure 3(a)), while a decrease in $\sigma$ results in broader PD (e.g., in Figure 3(c)). Based on the normalized PD in Figures 3(a)-3(c), the associated HL was drawn and shown in Figures 3(d)$3(\mathrm{f})$, respectively. Note that the normalization of PD helps conserving polarization saturation, which is useful when investigating a series of HL with different saturation levels.

In addition, the spiky PD in Figure 3(a) indicates that there is some particular field strength, that is, the coercive field, where hysteresis switching response becomes very strong. At this coercive point, the materials become more homogeneous, so the majority of the domains response to the switching field simultaneously. These pronounced responses have also been observed in various materials [13-15]. For instance, the peaked FORC distributions were shown to be a result of the amalgamation of ferromagnetic clusters and the dominance of wide-range ferromagnetic ordering in $\mathrm{La}_{1-x} \mathrm{Sr}_{x} \mathrm{CoO}_{3}$ [15]. As can be seen in Figure 3(d), the HL constructed from the sharp PD shows rapid changes of polarization around coercivity (high polarization derivatives).
On the other hand, the broader PD represents that materials respond to a wider range of external field. Therefore, the broad PD in Figures 3(b) and 3(c) results in the HL with gradual changes of polarization as seen in Figures 3(e) and 3(f). These characteristics can be found in material systems with less connected spins/system components or with high thermal fluctuation. For example, the broad FORC distributions, being found in porous Nb-PZT ceramics, address inhomogeneous ferroelectric switching $[13,16,17]$. Note that the broad PD is also found in thin films [18] or when frequencies of external field are very high [19]. In addition, this broad distribution can be used to indicate fatigue behavior via the FORC distribution of the hysteresis loops measured from PZT films [6]. This is as the fatigue decreases a capability of ferroelectric switching where the HL changes from a saturated loop to a minor loop (less saturated loop). The broad distribution feature therefore represents the loss of ferroelectric domain-wall mobility. Specifically, FORC distribution peak was found to be sharp for fresh sample but broad for fatigued sample. 


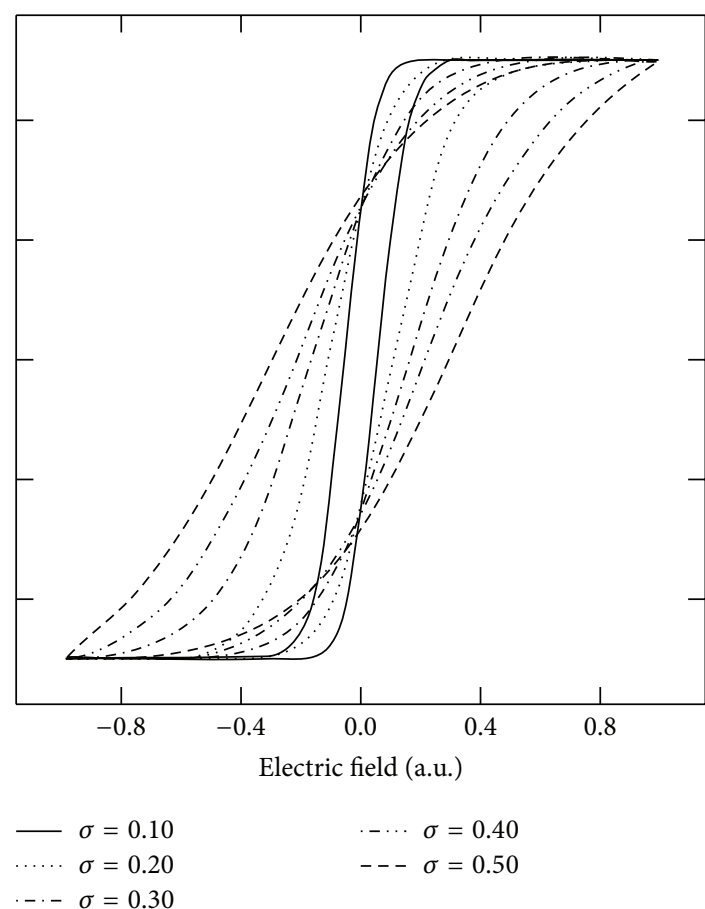

(a)

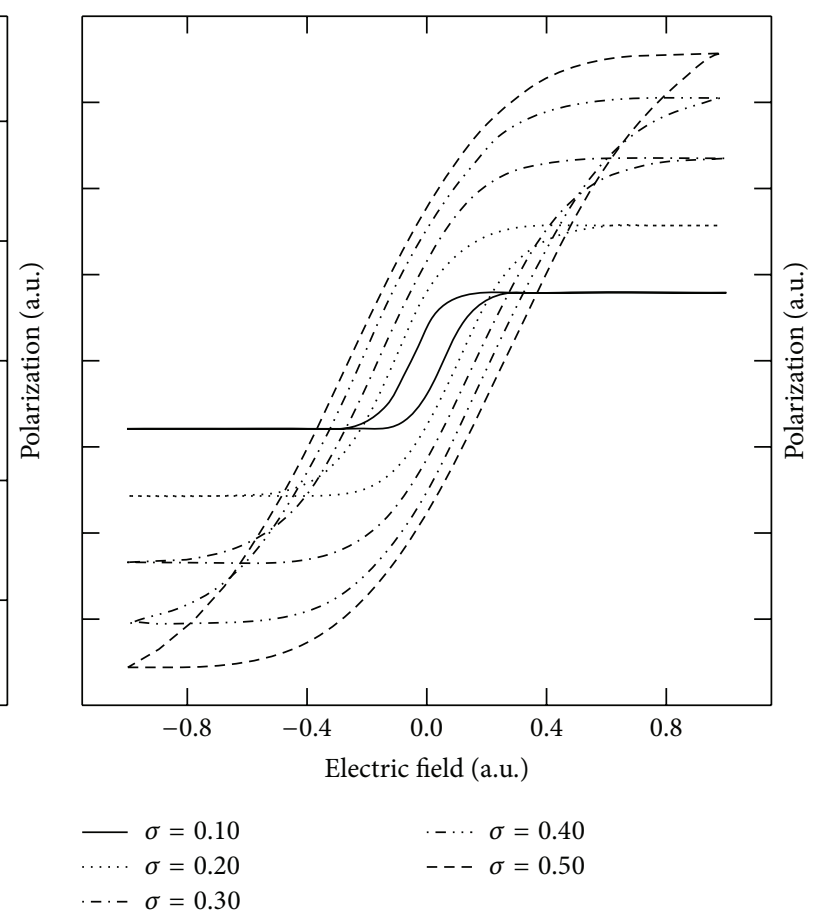

(b)

FiguRE 4: Hysteresis loops obtained from (a) normalized Preisach densities and (b) unnormalized Preisach densities for $\sigma=0.10-0.50$.

Therefore, an increase in $\sigma$ results in a more gradual reversal in HLs as can be seen in Figure 4. In other words, the increase in $\sigma$ in the Gaussian-defined PD represents an increase in switching availability in a wider range of external field and then results in evenly increment/decrement loops as seen in Figure 4(b). In similar, with the normalization of polarization saturations, HLs in Figure 4(a) also show sudden and slanted reversals at low and high $\sigma$, respectively.

4.2. Variation of the PD Peak Center. While the PD sharpness represents the dominance of switching responses around the PD center, the different location of the PD center, for example, $\left(x_{0}, y_{0}\right)$ in Figure 2(b), represents the different range of external field in which materials are active in switching. We investigated this by varying values of $\left(x_{0}, y_{0}\right)$ in (2) on the Preisach plane. Furthermore, due to the symmetric behavior of the HL, the PD center locates on the line $x_{0}=-y_{0}$, which yields a symmetric constrain between $E_{\beta}$ (increasing field) and $E_{\alpha}$ (decreasing field). Some of these PDs are shown in Figures 5(a)-5(c), and their constructed HLs are illustrated in Figures 5(d)-5(f).

It should be noted that, on the Preisach plane, the PD can be generally considered as being dominant at low field (low $E_{\alpha}$ and $E_{\beta}$ ) or at high field (high $E_{\alpha}$ and $E_{\beta}$ ) as shown in Figures 5(a)-5(c), respectively. While the PD with dominant switching at low field (e.g., Figure 5(a)) constructs a narrow loop as shown in Figure 5(d), the PD with dominant switching at the high field (e.g., Figure 5(c)) constructs a broad loop with large coercivity as shown in Figure 5(f). Similarly, the FORC distributions have been found to be peaked at either low field $[8,13,14,18,20,21]$ or high field $[16,17,22]$ corresponding to material characteristics and conditions of hysteresis measurement.

With $\sigma$ being fixed at 0.20 , the normalized PDs with different $\left(x_{0}, y_{0}\right)$ (in Figures 5(a)-5(c)) result in HLs as shown in Figures 5(d)-5(f) and 6(a). They show an increase in coercivity with increasing $y_{0}\left(\right.$ or $\left.\left|x_{0}\right|\right)$. For instance, from HLs constructed from normalized PDs, an increase in coercivity is obvious as shown in Figure 6(a). Therefore, the center of the PD peak $\left(x_{0}, y_{0}\right)$ can be used to indicate the field where materials tend to switch dominantly, that is, around the coercivity.

Nevertheless, the unnormalized PDs show some interesting behavior as HLs in Figure 6(b) show both increasing and decreasing of polarization saturations with increasing $y_{0}$. The reason for the increase in saturation is due to the move of PD from the upper-left corner to the center of the Preisach plane. The Gaussian PD surface then changes from a partial to a full one, which increases total weights of polarization switching included in the HL modeling. This eventually results in the increase in polarization saturations. On the opposite, the decrease in the saturation is caused by the move of PD center to where $x_{0}=y_{0}=0$ on the Preisach plane, where the Gaussian PD surface changes from a full to a partial Gaussian surface. Consequently, this results in a decrease in total contribution of polarization switching 

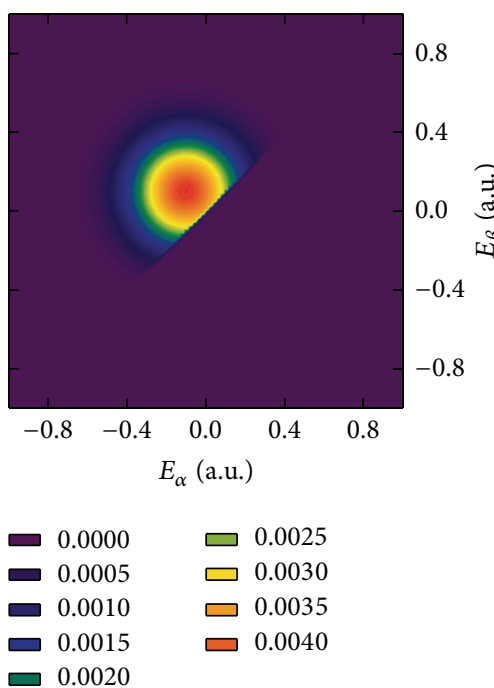

(a)

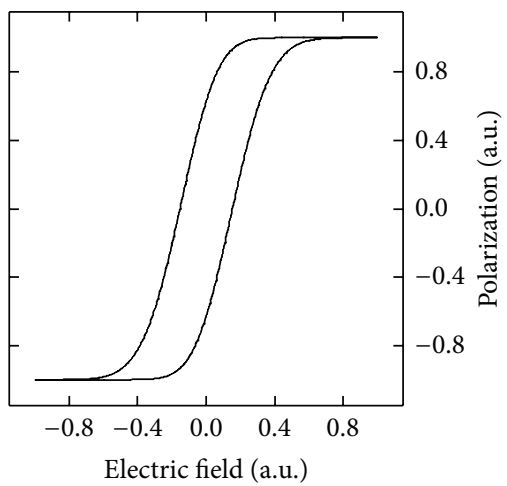

(d)

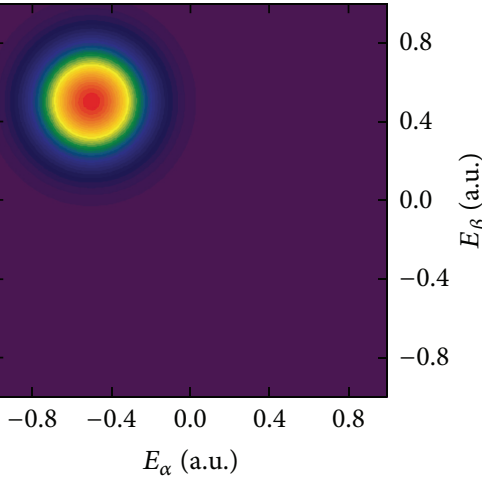

0.0000

0.0005

$\square 0.0020$

0.0025

$\square 0.0030$

$\square 0.0015$

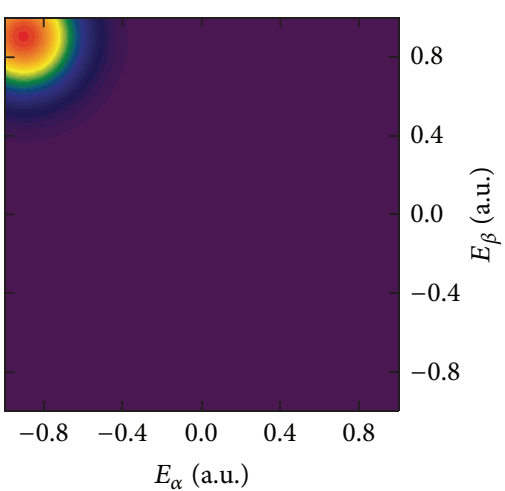

0.00
0.00
0.00
0.00

$\square .004$

$\square 0.005$

$\square 0.006$

(c)

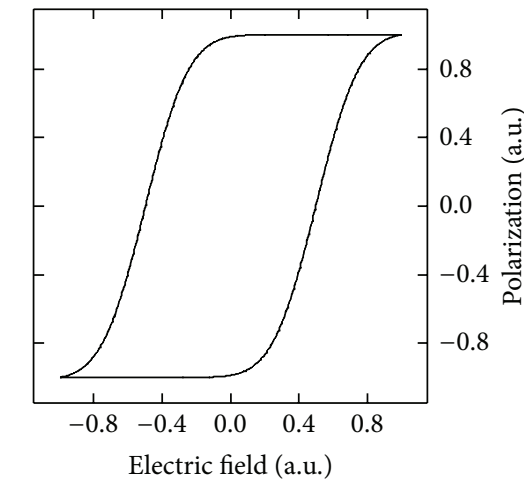

(e)

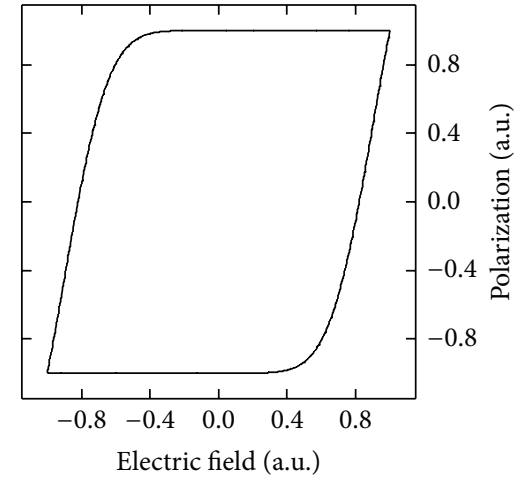

(f)

Figure 5: Gaussian-defined Preisach densities with $\sigma=0.20$ and centers located at (a) $y_{0}=0.10$, (b) $y_{0}=0.50$, and (c) $y_{0}=0.90$. Their associated hysteresis loops are shown in (d), (e), and (f), respectively.

included on the plane. These changes suggest that materials can be less responsive at very low and high fields. This is in fact related to loss of material response at very low field (where the field is too weak to drive the material) and at very high field (where the material is already at saturated state).

4.3. Splitting of the PD. Hysteresis loops with pinching characteristic or distorted loop are another feature that has been extensively investigated. Figure 7(a) shows PD with two main density peaks and the generated HL with pinching characteristic being plotted in Figure 7(b). The splitting peaks in the PD show that the material actively responses to more than one range of external fields. The consistency between the splitting peaks and the pinching feature was also found in various materials. For instance, in large-grained $\mathrm{BaTiO}_{3}$ ceramics, the defect dipoles cause pinched loops, where the dielectric loss was found to be contributed by peak separation in FORC distributions [8]. Also, the separated peaks in FORC distribution are found responsible for pinched HL in nanomagnets $[23,24]$. Consequently, the hysteresis pinching effect can be concurrently modeled using both the classical $\mathrm{PM}$ and the FORC distribution.

\section{Conclusion}

In this work, Preisach model was implemented to investigate the contributions of Preisach density to hysteresis reversals, where the Preisach densities were defined with threedimensional Gaussian distribution. The results show that the density pattern designs how polarization switching occurs in the hysteresis loop. Particularly, it was found that the sharpness of the densities represents how strong the switching is, while the density peak center represents a field range at which materials would response most actively. Also, the split of PD peaks details the pinching effect where material 

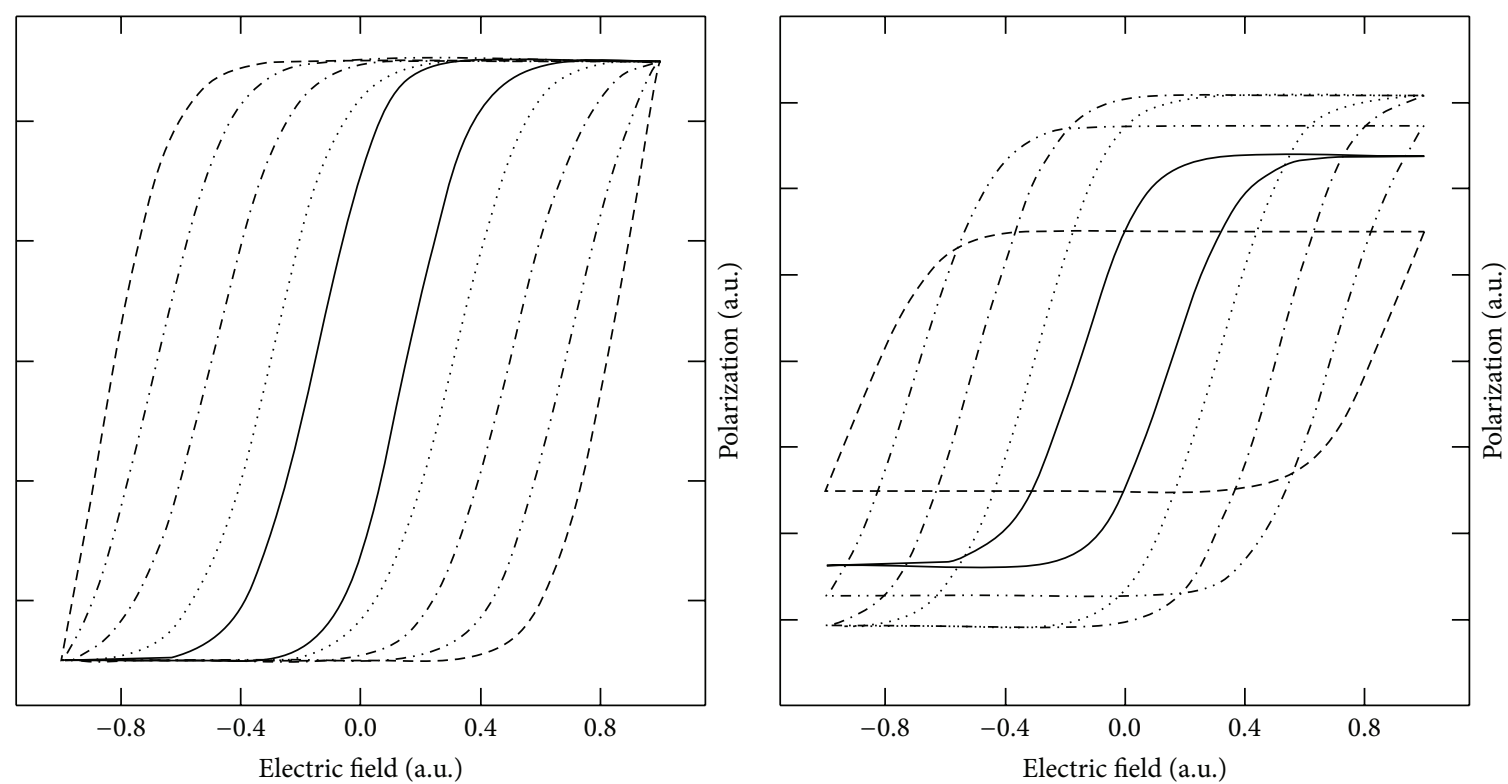

$$
\begin{array}{ll}
\text { — } & y_{0}=0.10 \\
\ldots \ldots & y_{0}=0.30 \\
\text {-. } \quad y_{0}=0.50
\end{array}
$$$$
\text { - - } y_{0}=0.70
$$$$
--y_{0}=0.90
$$

(a)

— $y_{0}=0.10$
$\ldots . \quad y_{0}=0.30$
_. $y_{0}=0.50$

$$
\text { - - . } y_{0}=0.70
$$$$
\text { - - } y_{0}=0.90
$$

(b)

Figure 6: Hysteresis loops obtained from (a) normalized Preisach densities and (b) unnormalized Preisach densities for $y_{0}=0.10-0.90$.
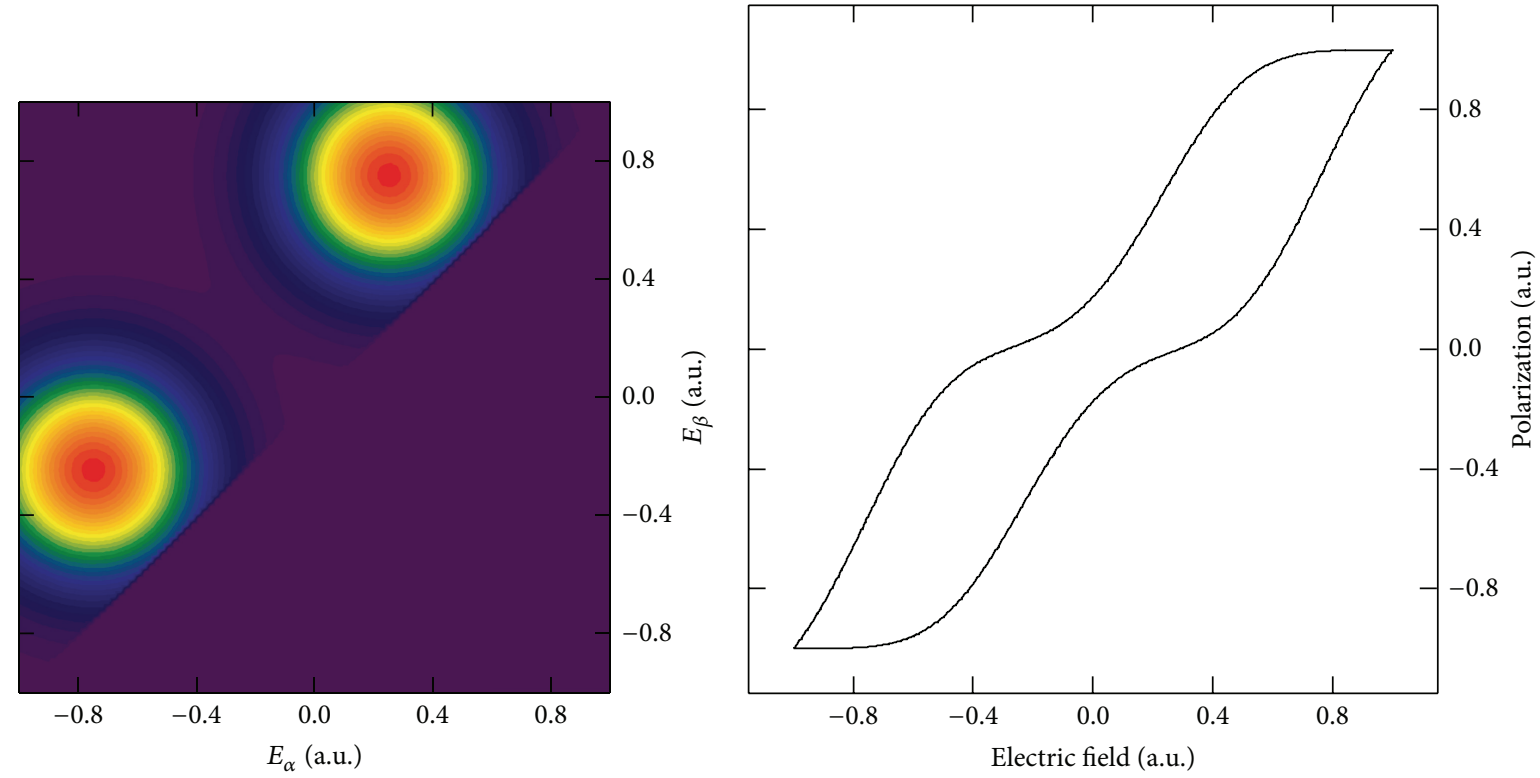

0.0000

0.0002

0.0004

0.0006 (a)

$$
\begin{aligned}
& \square 0.0008 \\
& \square 0.0010 \\
& \square \\
& 0.0012
\end{aligned}
$$

(b)

Figure 7: (a) Illustrations of two separate Preisach density peaks and (b) the associated pinched hysteresis loop. 
switching response is active in two separate ranges of the external field.

\section{References}

[1] Z. Yu, C. Ang, R. Guo, and A. S. Bhalla, "Ferroelectric-relaxor behavior of $\mathrm{Ba}\left(\mathrm{Ti}_{0.7} \mathrm{Zr}_{0.3}\right) \mathrm{O}_{3}$ ceramics," Journal of Applied Physics, vol. 92, no. 5, pp. 2655-2657, 2002.

[2] J. F. Scott, "Applications of modern ferroelectrics," Science, vol. 315, no. 5814, pp. 954-959, 2007.

[3] F. Preisach, "Über die magnetische Nachwirkung," Zeitschrift für Physik, vol. 94, no. 5-6, pp. 277-302, 1935.

[4] D. Bolten, U. Böttger, and R. Waser, "Reversible and irreversible polarization processes in ferroelectric ceramics and thin films," Journal of Applied Physics, vol. 93, no. 3, pp. 1735-1742, 2003.

[5] G. Robert, D. Damjanovic, and N. Setter, "Preisach modeling of ferroelectric pinched loops," Applied Physics Letters, vol. 77, no. 26, pp. 4413-4415, 2000.

[6] A. Stancu, D. Ricinschi, L. Mitoseriu, P. Postolache, and M. Okuyama, "First-order reversal curves diagrams for the characterization of ferroelectric switching," Applied Physics Letters, vol. 83, no. 18, pp. 3767-3769, 2003.

[7] C. R. Pike, A. P. Roberts, and K. L. Verosub, "Characterizing interactions in fine magnetic particle systems using first order reversal curves," Journal of Applied Physics, vol. 85, no. 9, pp. 6660-6667, 1999.

[8] I. Fujii, M. Ugorek, and S. Trolier-Mckinstry, "Grain size effect on the dielectric nonlinearity of $\mathrm{BaTiO}_{3}$ ceramics," Journal of Applied Physics, vol. 107, no. 10, Article ID 104116, 2010.

[9] C. I. Dobrotă and A. Stancu, "Mean field model for ferromagnetic nanowire arrays based on a mechanical analogy," Journal of Physics, vol. 25, no. 3, Article ID 035302, 2013.

[10] C. I. Dobrotă and A. Stancu, "What does a first-order reversal curve diagram really mean? A study case: array of ferromagnetic nanowires," Journal of Applied Physics, vol. 113, no. 4, pp. 043928-043911, 2013.

[11] I. Pintilie, I. Pasuk, G. A. Ibanescu et al., "The impact of the $\mathrm{Pb}(\mathrm{Zr}, \mathrm{Ti}) \mathrm{O}_{3}-\mathrm{ZnO}$ interface quality on the hysteretic properties of a metal-ferroelectric-semiconductor structure," Journal of Applied Physics, vol. 112, no. 10, pp. 104103-104108, 2012.

[12] I. D. Mayergoyz, "Mathematical models of hysteresis," Physical Review Letters, vol. 56, no. 15, pp. 1518-1521, 1986.

[13] M. Deluca, L. Stoleriu, L. P. Curecheriu et al., "High-field dielectric properties and Raman spectroscopic investigation of the ferroelectric-to-relaxor crossover in $\mathrm{BaSn}_{x} \mathrm{Ti}_{1-x} \mathrm{O}_{3}$ ceramics," Journal of Applied Physics, vol. 111, no. 8, Article ID 084102, 2012.

[14] M. Deluca, C. A. Vasilescu, A. C. Ianculescu et al., "Investigation of the composition-dependent properties of $\mathrm{BaTi}_{1-x} \mathrm{Zr}_{x} \mathrm{O}_{3}$ ceramics prepared by the modified Pechini method," Journal of the European Ceramic Society, vol. 32, no. 13, pp. 3551-3566, 2012.

[15] J. E. Davies, J. Wu, C. Leighton, and K. Liu, "Magnetization reversal and nanoscopic magnetic-phase separation in $\mathrm{La}_{1-x} \mathrm{Sr}_{x} \mathrm{CoO}_{3}$," Physical Review B, vol. 72, no. 13, Article ID 134419, 2005.

[16] D. Piazza, L. Stoleriu, L. Mitoseriu, A. Stancu, and C. Galassi, "Characterisation of porous PZT ceramics by first-order reversal curves (FORC) diagrams," Journal of the European Ceramic Society, vol. 26, no. 14, pp. 2959-2962, 2006.

[17] L. Stoleriu, A. Stancu, L. Mitoseriu, D. Piazza, and C. Galassi, "Analysis of switching properties of porous ferroelectric ceramics by means of first-order reversal curve diagrams," Physical Review B, vol. 74, no. 17, Article ID 174107, 2006.
[18] I. Fujii, E. Hong, and S. Trolier-Mckinstry, "Thickness dependence of dielectric nonlinearity of lead zirconate titanate films," IEEE Transactions on Ultrasonics, Ferroelectrics, and Frequency Control, vol. 57, no. 8, pp. 1717-1723, 2010.

[19] D. Ricinschi, L. Mitoseriu, A. Stancu, P. Postolache, and M. Okuyama, "Analysis of the switching characteristics of PZT films by first order reversal curve diagrams," Integrated Ferroelectrics, vol. 67, no. 1, pp. 103-115, 2004.

[20] I. Fujii, S. Trolier-Mckinstry, and C. Nies, "Effect of grain size on dielectric nonlinearity in model $\mathrm{BaTiO}_{3}$-based multilayer ceramic capacitors," Journal of the American Ceramic Society, vol. 94, no. 1, pp. 194-199, 2011.

[21] O. S. Ovchinnikov, S. Jesse, P. Bintacchit, S. Trolier-Mckinstry, and S. V. Kalinin, "Disorder identification in hysteresis data: recognition analysis of the random-bond-random-field ising model," Physical Review Letters, vol. 103, no. 15, Article ID 157203, 2009.

[22] O. Ovchinnikov, S. Jesse, S. Guo et al., "Local measurements of Preisach density in polycrystalline ferroelectric capacitors using piezoresponse force spectroscopy," Applied Physics Letters, vol. 96, no. 11, Article ID 112906, 2010.

[23] R. K. Dumas, C.-P. Li, I. V. Roshchin, I. K. Schuller, and K. Liu, "Magnetic fingerprints of sub- $100 \mathrm{~nm}$ Fe dots," Physical Review $B$, vol. 75, no. 13, Article ID 134405, 2007.

[24] R. K. Dumas, K. Liu, C.-P. Li, I. V. Roshchin, and I. K. Schuller, "Temperature induced single domain-vortex state transition in sub-100 nm Fe nanodots," Applied Physics Letters, vol. 91, no. 20, Article ID 202501, 2007. 

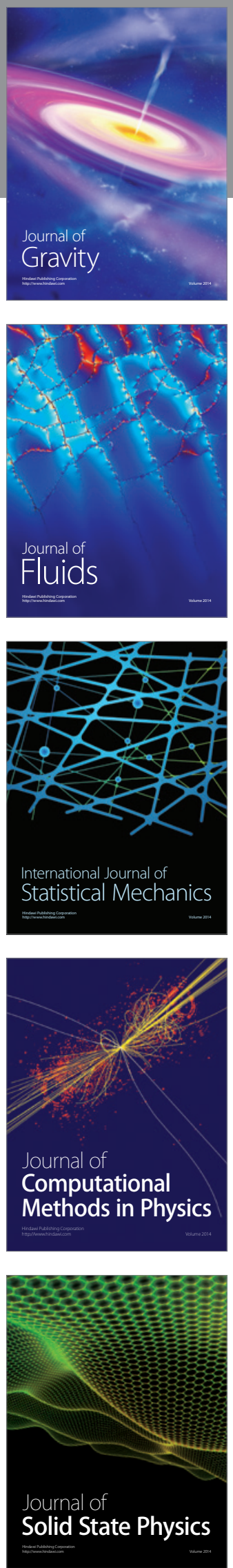

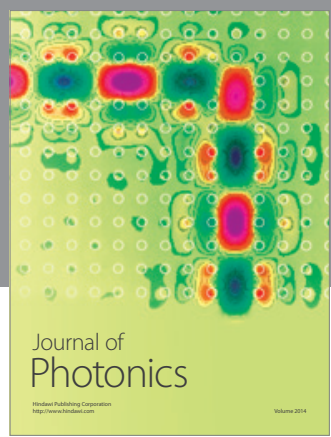

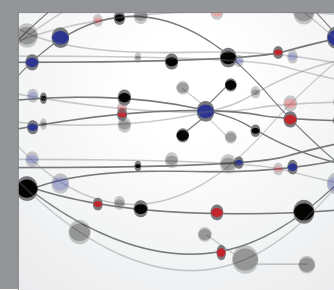

The Scientific World Journal

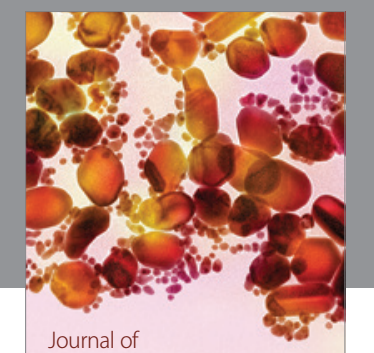

Soft Matter
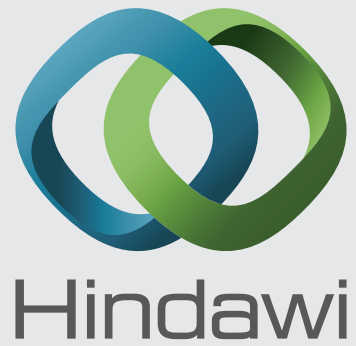

Submit your manuscripts at

http://www.hindawi.com
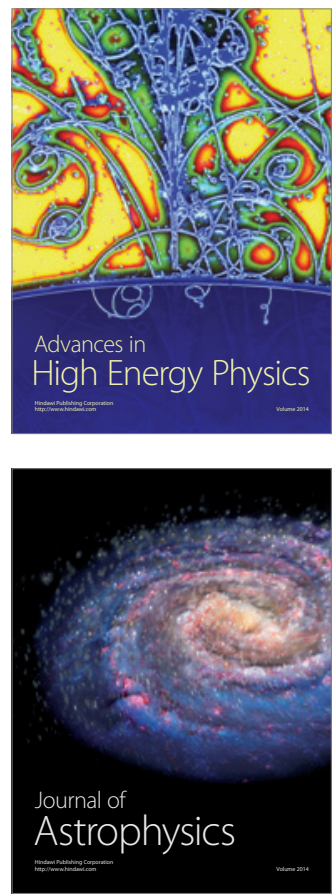
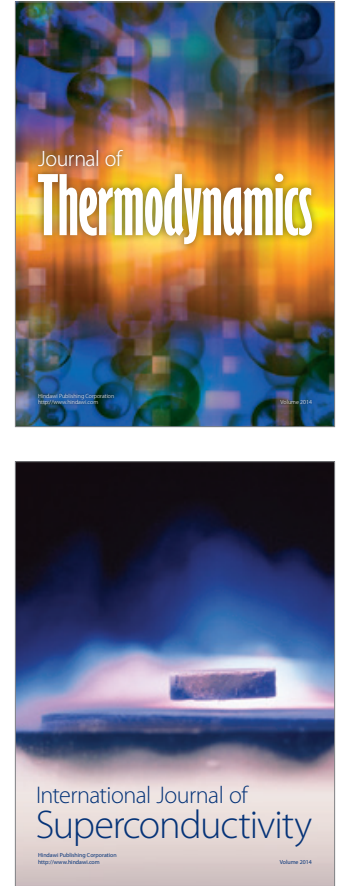
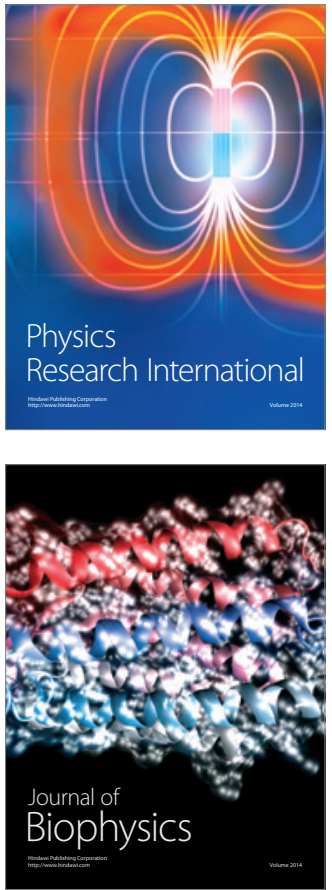
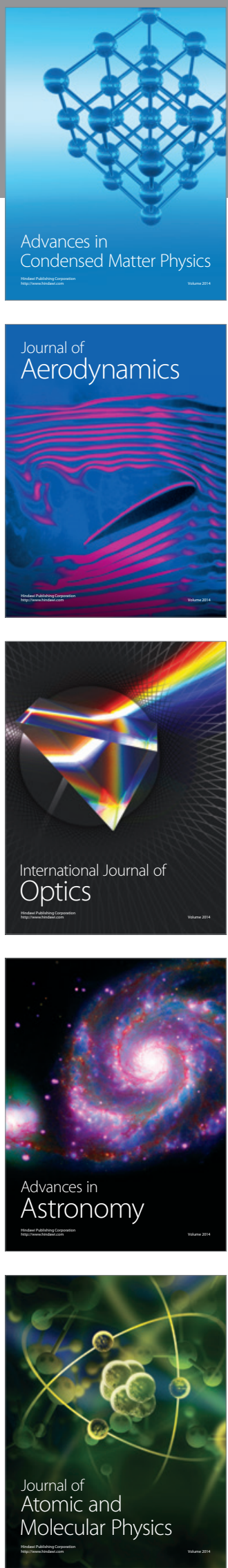